\title{
On Noisy Network Coding for a Gaussian Relay Chain Network with Correlated Noises
}

\author{
Lei Zhou and Wei Yu \\ Department of Electrical and Computer Engineering, \\ University of Toronto, Toronto, Ontario M5S 3G4, Canada \\ emails: \{zhoulei, weiyu\}@comm.utoronto.ca
}

\begin{abstract}
Noisy network coding, which elegantly combines the conventional compress-and-forward relaying strategy and ideas from network coding, has recently drawn much attention for its simplicity and optimality in achieving to within constant gap of the capacity of the multisource multicast Gaussian network. The constant-gap result, however, applies only to Gaussian relay networks with independent noises. This paper investigates the application of noisy network coding to networks with correlated noises. By focusing on a four-node Gaussian relay chain network with a particular noise correlation structure, it is shown that noisy network coding can no longer achieve to within constant gap to capacity with the choice of Gaussian inputs and Gaussian quantization. The cut-set bound of the relay chain network in this particular case, however, can be achieved to within half a bit by a simple concatenation of a correlation-aware noisy network coding strategy and a decode-and-forward scheme.
\end{abstract}

\section{INTRODUCTION}

The capacity region of the Gaussian relay network has been open for decades. Recently, the capacities of several relay networks with simple structures have been approximated to within constant number of bits. For example, for the threenode Gaussian relay channel, Avestimehr and Tse [1] showed that the decode-and-forward strategy achieves to within half a bit of the capacity; Chang, Chung, and Lee [2] proved that the compress-and-forward rate is within half a bit of the capacity, and the amplify-and-forward rate is within one bit.

In their breakthrough work, Avestimehr and Tse [1] further showed that, the capacity of the single-source singledestination Gaussian relay network in general can be achieved to within constant bits via a universal relaying scheme called quantize-map-and-forward (QMF). They also showed that, the gap to capacity is only related to the number of nodes in the network.

Parallel to Avestimehr and Tse's work, Lim, Kim, El Gamal, and Chung [3] proposed a noisy network coding strategy that naturally extends the conventional compress-and-forward scheme of Cover and El Gamal [4] and the classic network coding by Ahlswede, Cai, Li, and Yeung [5] to noisy networks. The main idea of noisy network coding is to derive an explicit expression of the achievable rate for each cut-set of the network. Then, by comparing with the cut-set upper bound, noisy network coding can be shown to achieve to within constant gap to the capacity of general multisource multicast Gaussian networks.

A key assumption made in both [1] and [3] is that the noises in the Gaussian relay network are independent with

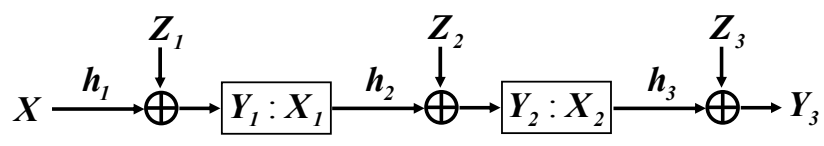

Fig. 1. A four-node Gaussian relay chain network

each other. This assumption may not hold in practical systems, where common interferences from other sources play a role. In this paper, we are interested in the following question. In the context of Gaussian relay networks with correlated noises, can noisy network coding achieve within constant bits to the capacity as well? This paper gives a negative answer by studying a four-node Gaussian chain network with correlated noises. It is shown that, in a certain scenario, the noisy-network-coding rate (with Gaussian input and Gaussian quantization) has an unbounded gap to the cut-set bound, whereas a concatenation of a modified correlation-aware noisy network coding strategy and a conventional decode-and-forward scheme achieves to within half a bit of the cut-set bound in this specific case.

\section{Channel Model}

The four-node Gaussian relay chain, as depicted in Fig. 1 consists of a source node, a destination node, and two relay nodes. The source communicates with the destination with the help from the two relays in between. Information passes from the source to the neighboring relay, and to the next, then finally to the destination. The input-output relationship can be described as follows:

$$
\begin{aligned}
& Y_{1}=h_{1} X+Z_{1}, \\
& Y_{2}=h_{2} X_{1}+Z_{2}, \\
& Y_{3}=h_{3} X_{2}+Z_{3} .
\end{aligned}
$$

Without loss of generality, assume that the transmit power of all nodes are normalized to one, and the variances of the receiver noises are also normalized to one, i.e., $Z_{i} \sim \mathcal{N}(0,1)$. The receiver noises are i.i.d. in time, but the noises $\left[Z_{1}, Z_{2}, Z_{3}\right]$ are correlated with the following correlation matrix:

$$
K_{Z}=\left[\begin{array}{ccc}
1 & \rho_{12} & \rho_{13} \\
\rho_{12} & 1 & \rho_{23} \\
\rho_{13} & \rho_{23} & 1
\end{array}\right],
$$

where $K_{z}$ is positive semidefinite and $\rho_{i j}$ is the correlation coefficient between $Z_{i}$ and $Z_{j}$. Note that the relay operation must be causal in time. 


\section{Suboptimality of the Noisy Network Coding}

We begin by showing that using noisy network coding with the choices of Gaussian inputs and Gaussian quantization noises, the gap between the achievable rate and the cut-set bound can be unbounded for a Gaussian relay chain network with a certain noise correlation structure. First, an upper bound to the cut-set bound of the four-node relay chain can be computed as follows:

$$
\begin{aligned}
& \max _{p\left(x, x_{1}, x_{2}\right)} \min \left\{I\left(X ; Y_{1} Y_{2} Y_{3} \mid X_{1} X_{2}\right), I\left(X X_{1} ; Y_{2} Y_{3} \mid X_{2}\right),\right. \\
& \left.I\left(X X_{1} X_{2} ; Y_{3}\right), I\left(X X_{2} ; Y_{1} Y_{3} \mid X_{1}\right)\right\} \\
& \leq \min \left\{\max I\left(X ; Y_{1} Y_{2} Y_{3} \mid X_{1} X_{2}\right), \max I\left(X X_{1} ; Y_{2} Y_{3} \mid X_{2}\right)\right. \text {, } \\
& \left.\max I\left(X X_{1} X_{2} ; Y_{3}\right), \max I\left(X X_{2} ; Y_{1} Y_{3} \mid X_{1}\right)\right\} \\
& =\min \left\{\bar{C}\left(\mathcal{S}_{1}\right), \bar{C}\left(\mathcal{S}_{2}\right), \bar{C}\left(\mathcal{S}_{3}\right), \bar{C}\left(\mathcal{S}_{4}\right)\right\} \\
& \triangleq \bar{C}
\end{aligned}
$$

where the cut-sets are defined as $\mathcal{S}_{1}=\{X\}, \mathcal{S}_{2}=\left\{X, X_{1}\right\}$, $\mathcal{S}_{3}=\left\{X, X_{1}, X_{2}\right\}$, and $\mathcal{S}_{4}=\left\{X, X_{2}\right\}$, and $\bar{C}\left(\mathcal{S}_{i}\right), i=$ $1,2,3,4$ are the four cut-set upper bounds, which can be calculated as

$$
\begin{aligned}
\bar{C}\left(\mathcal{S}_{1}\right) & =\max I\left(X ; Y_{1} Y_{2} Y_{3} \mid X_{1} X_{2}\right) \\
& =\frac{1}{2} \log \left(1+\frac{\left(1-\rho_{23}^{2}\right) h_{1}^{2}}{\left|K_{Z}\right|}\right),
\end{aligned}
$$

and

$$
\begin{aligned}
\bar{C}\left(\mathcal{S}_{2}\right) & =\max I\left(X X_{1} ; Y_{2} Y_{3} \mid X_{2}\right) \\
& =\frac{1}{2} \log \left(1+\frac{h_{2}^{2}}{1-\rho_{23}^{2}}\right),
\end{aligned}
$$

and

$$
\begin{aligned}
\bar{C}\left(\mathcal{S}_{3}\right) & =\max I\left(X X_{1} X_{2} ; Y_{3}\right) \\
& =\frac{1}{2} \log \left(1+h_{3}^{2}\right),
\end{aligned}
$$

and

$$
\begin{aligned}
\bar{C}\left(S_{4}\right) & =\max I\left(X X_{2} ; Y_{1} Y_{3} \mid X_{1}\right) \\
& =\frac{1}{2} \log \left(1+\frac{h_{1}^{2}+h_{3}^{2}+h_{1}^{2} h_{3}^{2}}{1-\rho_{13}^{2}}\right) \\
& \geq \bar{C}\left(\mathcal{S}_{3}\right),
\end{aligned}
$$

which is redundant.

The main point of noisy network coding is that an achievable rate can be derived for each of the cut-sets $\mathcal{S}_{1}, \mathcal{S}_{2}$, and $\mathcal{S}_{3}$ using a generalization of the compress-and-forward scheme. For convenience, we state the achievable rates as follows.

Theorem 1 (Noisy Network Coding Theorem [3]). Let $\mathcal{D}=$ $\mathcal{D}_{1}=\mathcal{D}_{2}=\cdots=\mathcal{D}_{N}$. A rate tuple $\left(R_{1}, \cdots, R_{N}\right)$ is achievable for the DMN $p\left(y^{N} \mid x^{N}\right)$ if there exists some joint pmf $p(q) \prod_{k=1}^{N} p\left(x_{k} \mid q\right) p\left(\hat{y}_{k} \mid y_{k}, x_{k}, q\right)$ such that

$$
\begin{array}{r}
R(\mathcal{S})<\min _{d \in \mathcal{S}^{c} \cap \mathcal{D}} I\left(X(\mathcal{S}) ; \hat{Y}\left(\mathcal{S}^{c}\right), Y_{d} \mid X\left(\mathcal{S}^{c}\right), Q\right) \\
-I\left(Y(\mathcal{S}) ; \hat{Y}(\mathcal{S}) \mid X^{N}, \hat{Y}\left(\mathcal{S}^{c}\right), Y_{d}, Q\right)
\end{array}
$$

for all cutsets $\mathcal{S} \subseteq[1: N]$ with $S^{c} \cap \mathcal{D} \neq \emptyset$, where $R(\mathcal{S})=$ $\sum_{k \in \mathcal{S}} R_{k}$.

Although the quantization in the above noisy network coding theorem can in theory have arbitrary distributions, Gaussian inputs and Gaussian quantization noises are usually adopted for Gaussian networks [?] [6], and are shown to achieve constant gap to capacity for networks with uncorrelated noises [3]. Thus, this paper follows the same choice, i.e.,

$$
\hat{Y}_{i}=Y_{i}+\hat{Z}_{i}
$$

where the quantization noise $\hat{Z}_{i} \sim \mathcal{N}\left(0, \mathrm{q}_{i}\right)$ is independent with everything else. Now applying the noisy network coding theorem with Gaussian inputs and Gaussian quantization noises, the following achievable rates for cut-sets $\mathcal{S}_{1}, \mathcal{S}_{2}$, and (1) $\mathcal{S}_{3}$ can be derived:

$$
\begin{aligned}
R\left(\mathcal{S}_{1}\right) & =I\left(X ; \hat{Y}_{1} \hat{Y}_{2} Y_{3} \mid X_{1} X_{2}\right) \\
& =h\left(\begin{array}{r}
h_{1} X+Z_{1}+\hat{Z}_{1} \\
Z_{2}+\hat{Z}_{2} \\
Z_{3}
\end{array}\right)-h\left(\begin{array}{r}
Z_{1}+\hat{Z}_{1} \\
Z_{2}+\hat{Z}_{2} \\
Z_{3}
\end{array}\right) \\
& =\frac{1}{2} \log \left(1+\frac{\left(1+\mathrm{q}_{2}-\rho_{23}^{2}\right) h_{1}^{2}}{\left|K_{\beta}\right|}\right),
\end{aligned}
$$

where

$$
\left|K_{\beta}\right|=\left|\begin{array}{ccc}
1+\mathrm{q}_{1} & \rho_{12} & \rho_{13} \\
\rho_{12} & 1+\mathrm{q}_{2} & \rho_{23} \\
\rho_{13} & \rho_{23} & 1
\end{array}\right|,
$$

and

$$
\begin{aligned}
R\left(\mathcal{S}_{2}\right)= & I\left(X X_{1} ; \hat{Y_{2}} Y_{3} \mid X_{2}\right)-I\left(Y_{1} ; \hat{Y}_{1} \mid X X_{1} X_{2} \hat{Y}_{2} Y_{3}\right) \\
= & \frac{1}{2} \log \left(1+\frac{h_{2}^{2}}{1+\mathrm{q}_{2}-\rho_{23}^{2}}\right) \\
& \quad-\frac{1}{2} \log \left(1+\frac{1-\rho_{13}^{2}}{\mathrm{q}_{1}}\right) .
\end{aligned}
$$

and

$$
\begin{aligned}
R\left(\mathcal{S}_{3}\right)= & I\left(X X_{1} X_{2} ; Y_{3}\right)-I\left(Y_{1} Y_{2} ; \hat{Y}_{1} \hat{Y}_{2} \mid X X_{1} X_{2} Y_{3}\right) \\
= & \frac{1}{2} \log \left(1+h_{3}^{2}\right) \\
& -\frac{1}{2} \log \frac{\left|\begin{array}{cc}
1-\rho_{13}^{2}+\mathrm{q}_{1} & \rho_{12}-\rho_{13} \rho_{23} \\
\rho_{12}-\rho_{13} \rho_{23} & 1-\rho_{23}^{2}+\mathrm{q}_{2}
\end{array}\right|}{\mathrm{q}_{1} \mathrm{q}_{2}} .
\end{aligned}
$$

The achievable rate is then upper bounded by the minimum of the three:

$$
R \leq \min \left\{R\left(\mathcal{S}_{1}\right), R\left(\mathcal{S}_{2}\right), R\left(\mathcal{S}_{3}\right)\right\} .
$$

Now, consider a special scenario when the noise $Z_{3}$ is independent with both $Z_{1}$ and $Z_{2}$, and channel strengths $h_{2}^{2}$ and $h_{3}^{2}$ scale with $h_{1}^{2}$, i.e.,

$$
\rho_{13}=\rho_{23}=0,
$$

and

$$
h_{2}^{2}=h_{3}^{2}=\frac{h_{1}^{2}}{1-\rho_{12}^{2}} .
$$


This special setting gives us the following cut-set bounds:

$$
\bar{C}\left(\mathcal{S}_{1}\right)=\bar{C}\left(\mathcal{S}_{2}\right)=\bar{C}\left(\mathcal{S}_{3}\right)=\frac{1}{2} \log \left(1+\frac{h_{1}^{2}}{1-\rho_{12}^{2}}\right),
$$

and the following achievable rates for cut-sets $\mathcal{S}_{1}$ to $\mathcal{S}_{3}$ :

$$
\begin{aligned}
R\left(\mathcal{S}_{1}\right)= & \frac{1}{2} \log \left(1+\frac{h_{1}^{2}}{1+\mathrm{q}_{1}-\frac{\rho_{12}^{2}}{1+\mathrm{q}_{2}}}\right), \\
R\left(\mathcal{S}_{2}\right)= & \frac{1}{2} \log \left(1+\frac{h_{1}^{2}}{\left(1-\rho_{12}^{2}\right)\left(1+\mathrm{q}_{2}\right)}\right) \\
& -\frac{1}{2} \log \left(1+\frac{1}{\mathrm{q}_{1}}\right), \\
R\left(\mathcal{S}_{3}\right)= & \frac{1}{2} \log \left(1+\frac{h_{1}^{2}}{1-\rho_{12}^{2}}\right) \\
& -\frac{1}{2} \log \left(1+\frac{\mathrm{q}_{1}+\mathbf{q}_{2}+1-\rho_{12}^{2}}{\mathbf{q}_{1} \mathbf{q}_{2}}\right) .
\end{aligned}
$$

Next, we show that, the gaps $\bar{C}\left(\mathcal{S}_{1}\right)-R\left(\mathcal{S}_{1}\right), \bar{C}\left(\mathcal{S}_{2}\right)-$ $R\left(\mathcal{S}_{2}\right), \bar{C}\left(\mathcal{S}_{3}\right)-R\left(\mathcal{S}_{3}\right)$ cannot be made all finite when $\rho_{12}^{2} \rightarrow$ 1 . First, the gap on the cut-set $\mathcal{S}_{1}$ is given by

$$
\begin{aligned}
\Delta\left(\mathcal{S}_{1}\right)= & \bar{C}\left(\mathcal{S}_{1}\right)-R\left(\mathcal{S}_{1}\right) \\
= & \frac{1}{2} \log \left(1+\frac{h_{1}^{2}}{1-\rho_{12}^{2}}\right) \\
& -\frac{1}{2} \log \left(1+\frac{h_{1}^{2}}{1+\mathrm{q}_{1}-\frac{\rho_{12}^{2}}{1+\mathrm{q}_{2}}}\right),
\end{aligned}
$$

and the gap on the cut-set $\mathcal{S}_{2}$ is lower bounded by

$$
\begin{aligned}
\Delta\left(\mathcal{S}_{2}\right)= & \bar{C}\left(\mathcal{S}_{2}\right)-R\left(\mathcal{S}_{2}\right) \\
= & \frac{1}{2} \log \left(1+\frac{h_{1}^{2}}{1-\rho_{12}^{2}}\right)+\frac{1}{2} \log \left(1+\frac{1}{\mathrm{q}_{1}}\right) \\
& -\frac{1}{2} \log \left(1+\frac{h_{1}^{2}}{\left(1-\rho_{12}^{2}\right)\left(1+\mathrm{q}_{2}\right)}\right) \\
\geq & \frac{1}{2} \log \left(1+\frac{1}{\mathrm{q}_{1}}\right),
\end{aligned}
$$

and the gap on the cut-set $\mathcal{S}_{3}$ is lower bounded by the same number as well, i.e.,

$$
\begin{aligned}
\Delta\left(\mathcal{S}_{3}\right) & =\bar{C}\left(\mathcal{S}_{3}\right)-R\left(\mathcal{S}_{3}\right) \\
& =\frac{1}{2} \log \left(1+\frac{\mathrm{q}_{1}+\mathrm{q}_{2}+1-\rho_{12}^{2}}{\mathrm{q}_{1} \mathrm{q}_{2}}\right) \\
& \geq \frac{1}{2} \log \left(1+\frac{1}{\mathrm{q}_{1}}\right) .
\end{aligned}
$$

Now, since

$$
\bar{C}-R \geq \max \left\{\Delta\left(\mathcal{S}_{1}\right), \Delta\left(\mathcal{S}_{2}\right), \Delta\left(\mathcal{S}_{3}\right)\right\},
$$

in order to make $\bar{C}-R$ finite, all three gaps have to be upper bounded by a finite number. Inspecting the gap of $\Delta\left(\mathcal{S}_{1}\right)$ in (16), in order to make it finite when $\rho_{12} \rightarrow \infty$, both $\mathrm{q}_{1}$ and $\mathrm{q}_{2}$ have to go to zero. However, in this case, $\Delta\left(\mathcal{S}_{2}\right)$ and $\Delta\left(\mathcal{S}_{3}\right)$ are apparently unbounded. Therefore, in the scenario of $(13)$ and 14 , as $\rho_{12}^{2}$ goes to 1 , it is impossible to keep all three gaps $\Delta\left(\mathcal{S}_{1}\right), \Delta\left(\mathcal{S}_{2}\right)$, and $\Delta\left(\mathcal{S}_{3}\right)$ finite simultaneously. As a consequence, for the four-node Gaussian chain network with correlated noises, the noisy network coding achievable rate with the choice of Gaussian inputs and Gaussian quantization noises has an unbounded gap to the cut-set upper bound.

\section{An Optimal CONCATENATED Scheme}

It is known that the cut-set upper bound is not always tight for the relay channel [7], [8], but for the four-node Gaussian chain network, does the cut-set bound have an infinite gap to capacity? Or, is it the noisy networking coding achievable rate that has an infinite gap to capacity? To answer this question, we show in the following that the cut-set bound (15) can actually be achieved to within half a bit for this four-node relay network with the particular noise correlation structure (13) by a simple concatenation of a correlation-aware noisy network coding strategy and a conventional decode-andforward scheme. This justifies the suboptimality of the noisy network coding for Gaussian relay networks with correlated noises.

Inspecting the structure of the four-node relay network in Fig. 1 and the special correlation structure (13), it is easy to see that the last node is essentially independent of the first three nodes in this example. Now the first three nodes (from the source node $X$ to the second relay node $Y_{2}$ ) forms a three-node Gaussian relay channel, so we can apply the noisy network coding theorem just to the first three nodes. With the source message decoded at $Y_{2}$, the second relay node can then reencode the source information and forward to the destination $Y_{3}$. With $Y_{1}$ serving as a noisy-network-coding type of relay and $Y_{2}$ serving as a decode-and-forward type of relay, this is essentially a concatenation of the noisy network coding and the decode-and-forward scheme. The achievable rate of this concatenated scheme can be derived as follows.

In the first relaying stage where $Y_{1}$ serves as a noisynetwork-coding type of relay, according to the noisy network coding theorem [3, Theorem 1], $Y_{2}$ can decode the source message if the following rate is satisfied:

$$
\begin{gathered}
R \leq \min \left\{I\left(X, X_{1} ; Y_{2}\right)-I\left(Y_{1} ; \hat{Y}_{1} \mid X, X_{1}, Y_{2}\right),\right. \\
\left.I\left(X ; Y_{2}, \hat{Y}_{1} \mid X_{1}\right)\right\}
\end{gathered}
$$

for some distribution

$$
p\left(x, x_{1}, y_{1}, \hat{y}_{1}\right)=p(x) p\left(x_{1}\right) p\left(y_{1} \mid x, x_{1}\right) p\left(\hat{y}_{1} \mid x_{1}, y_{1}\right) .
$$

Substituting Gaussian inputs $X \sim \mathcal{N}(0,1), X_{1} \sim \mathcal{N}(0,1)$, and Gaussian quantization signal $\hat{Y}_{1}=Y_{1}+\hat{Z}_{1}$, where $\hat{Z}_{1} \sim$ $\mathcal{N}\left(0, q_{1}\right)$ is independent with everything else, we have the following achievable rate in the first stage:

$$
\begin{aligned}
R \leq \min \{ & \frac{1}{2} \log \left(1+\frac{h_{1}^{2}}{1-\rho_{12}^{2}}\right)-\frac{1}{2} \log \left(1+\frac{\mathbf{q}_{1}}{1-\rho_{12}^{2}}\right) \\
& \left.\frac{1}{2} \log \left(1+h_{2}^{2}\right)-\frac{1}{2} \log \left(1+\frac{1-\rho_{12}^{2}}{\mathbf{q}_{1}}\right)\right\} .
\end{aligned}
$$

Next, with the source message decoded at $Y_{2}$, the second relay node acts as a decode-and-forward type of relay, which 
re-encodes and forwards the source message to the destination $Y_{3}$ through the Gaussian channel of channel gain $h_{3}$. The destination can successfully decode the source message if

$$
R \leq \frac{1}{2} \log \left(1+h_{3}^{2}\right) .
$$

Combining the above rate constraints (21) and 22) gives us the following achievable rate by the concatenated scheme:

$$
\begin{aligned}
R \leq \min \{ & \frac{1}{2} \log \left(1+\frac{h_{1}^{2}}{1-\rho_{12}^{2}}\right)-\frac{1}{2} \log \left(1+\frac{\mathrm{q}_{1}}{1-\rho_{12}^{2}}\right), \\
& \frac{1}{2} \log \left(1+h_{2}^{2}\right)-\frac{1}{2} \log \left(1+\frac{1-\rho_{12}^{2}}{\mathrm{q}_{1}}\right) \\
& \left.\frac{1}{2} \log \left(1+h_{3}^{2}\right)\right\} .
\end{aligned}
$$

Comparing the above achievable rate with the cut-set upper bound with $\rho_{12}$ and $\rho_{23}$ set to zero:

$$
\begin{aligned}
\bar{C}=\min \{ & \frac{1}{2} \log \left(1+\frac{h_{1}^{2}}{1-\rho_{12}^{2}}\right), \frac{1}{2} \log \left(1+h_{2}^{2}\right), \\
& \left.\frac{1}{2} \log \left(1+h_{3}^{2}\right)\right\},
\end{aligned}
$$

we have the difference upper bounded by

$\bar{C}-R \leq \max \left\{\frac{1}{2} \log \left(1+\frac{\mathbf{q}_{1}}{1-\rho_{12}^{2}}\right), \frac{1}{2} \log \left(1+\frac{1-\rho_{12}^{2}}{\mathbf{q}_{1}}\right)\right\}$

It is easy to see that the first term monotonically increases with $\mathrm{q}_{1}$ while the second term monotonically decreases. As a result, to minimize the maximum of the two terms, we need

$$
\frac{1}{2} \log \left(1+\frac{1-\rho_{12}^{2}}{\mathbf{q}_{1}^{*}}\right)=\frac{1}{2} \log \left(1+\frac{\mathrm{q}_{1}^{*}}{1-\rho_{12}^{2}}\right),
$$

which results in the optimal correlation-aware quantization level $\mathrm{q}_{1}^{*}=1-\rho_{12}^{2}$. Substituting $\mathrm{q}_{1}^{*}$ into 25 gives us $\bar{C}-R<$ $\frac{1}{2}$. Therefore, for the four-node Gaussian chain network as shown in Fig. 11 in the scenario where $\rho_{13}=\rho_{23}=0$, the cut-set upper bound can be achieved to within constant gap.

Fig. 2 shows a numerical example for comparing noisy network coding and the concatenated scheme. In the simulation, we let $h_{1}^{2}=20 \mathrm{~dB}$ and all other channel parameters are set to satisfy (13) and (14). For the quantization parameters, we choose the optimal quantization level $\mathrm{q}_{1}=1-\rho_{12}^{2}$ and let $\mathrm{q}_{2}=1$. As can be seen from the figure, when $\rho_{12}$ approaches to +1 or -1 , both the cut-set upper bound and the achievable rate by the concatenated scheme go to infinity. However, the achievable rate by the noisy network coding scheme remains finite, making the gap to the cut-set bound unbounded.

\section{CONCLUSION}

This paper studies the optimality of the noisy network coding for a four-node Gaussian chain network with correlated noises. It is shown that, under a certain noise correlation structure, noisy network coding with Gaussian inputs and Gaussian quantization noises has an infinite gap to the cut-set upper bound. But, the upper bound can be achieved to within half a bit in this specific case by a simple concatenation of a

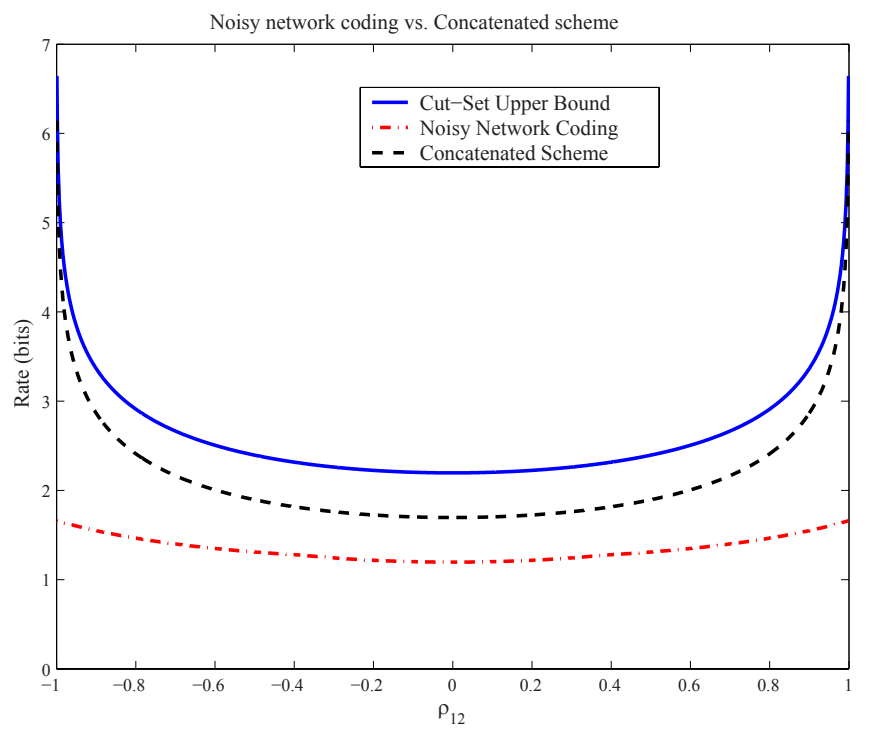

Fig. 2. Noisy network coding vs. concatenated scheme

correlation-aware noisy network coding strategy and a decodeand-forward scheme.

\section{REFERENCES}

[1] S. Avestimehr, S. Diggavi, and D. Tse, "Wireless network information flow: a deterministic approach," Submitted to IEEE Trans. Inf. Theory, 2009.

[2] W. Chang, S.-Y. Chung, and Y. H. Lee, "Gaussian relay channel capacity to within a fixed number of bits," 2010. [Online]. Available: http://arxiv.org/abs/1011.5065

[3] S.-Y. Lim, Y.-H. Kim, A. El Gamal, and S.-Y. Chung, "Noisy network coding," Submitted to IEEE Trans. Inf. Theory, 2010.

[4] T. M. Cover and A. El Gamal, "Capacity theorems for the relay channel," IEEE Trans. Inf. Theory, vol. 25, no. 5, pp. 572-584, Sep. 1979.

[5] R. Ahlswede, N. Cai, R. Li, and R. W. Yeung, "Network information flow," IEEE Trans. Inf. Theory, vol. 46, pp. 1004 -1016, Jul 2000.

[6] L. Zhou and W. Yu, "Gaussian z-interference channel with a relay link: achievability region and asymptotic sum capacity," 2010. [Online]. Available: http://arxiv.org/abs/1006.5087

[7] M. Aleksic, P. Razaghi, and W. Yu, "Capacity of a class of modulo-sum relay channels," IEEE Trans. Inf. Theory, vol. 55, no. 3, pp. 921-930, Mar. 2009.

[8] Z. Zhang, "Partial converse for a relay channel," IEEE Trans. Inf. Theory, vol. 34 , no. 5, pp. 1106-1110, Sep. 1988. 